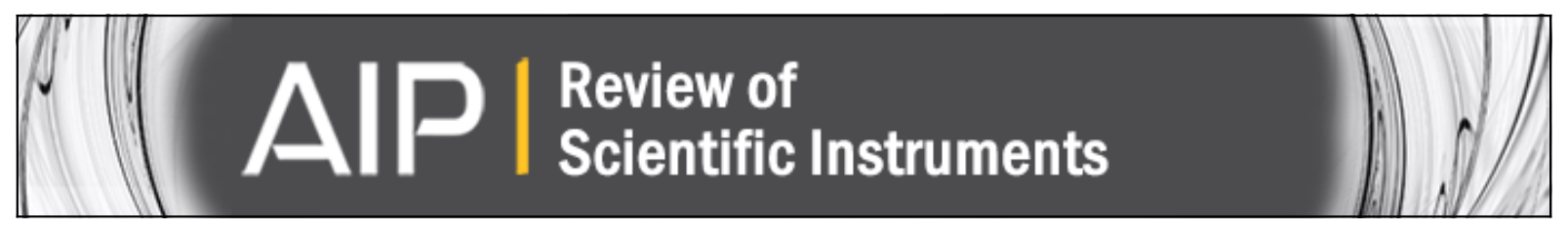

\title{
Metal-ceramic laminate composite magnetoelectric gradiometer
}

V. Bedekar, M. I. Bichurin, S. N. Ivanov, Y. J. Pukinski, and S. Priya

Citation: Review of Scientific Instruments 81, 033906 (2010); doi: 10.1063/1.3354985

View online: http://dx.doi.org/10.1063/1.3354985

View Table of Contents: http://scitation.aip.org/content/aip/journal/rsi/81/3?ver=pdfcov

Published by the AIP Publishing

\section{AlP Re-register for Table of Content Alerts}




\title{
Metal-ceramic laminate composite magnetoelectric gradiometer
}

\author{
V. Bedekar, ${ }^{1}$ M. I. Bichurin, ${ }^{2}$ S. N. Ivanov,${ }^{2}$ Y. J. Pukinski, ${ }^{2}$ and S. Priya ${ }^{1, a)}$ \\ ${ }^{1}$ Materials Science and Engineering, Center for Energy Harvesting Materials and Systems (CEHMS), \\ Virginia Tech, Blacksburg, Virginia 24061, USA \\ ${ }^{2}$ Novgorod State University, Veliky Novgorod 173003, Russia
}

(Received 14 January 2010; accepted 13 February 2010; published online 16 March 2010)

\begin{abstract}
Gradiometer resembles in functionality a magnetic field sensor where it measures the magnetic field gradient and its sensitivity is determined by the ability to quantify differential voltage change with respect to a reference value. Magnetoelectric (ME) gradiometer designed in this study is based upon the nickel $(\mathrm{Ni})-\mathrm{Pb}(\mathrm{Zr}, \mathrm{Ti}) \mathrm{O}_{3}(\mathrm{PZT})$ composites and utilizes the ring-dot piezoelectric transformer structure working near the resonance as the basis. The samples had the ring-dot electrode pattern printed on the top surface of PZT, where ring acts as the input while dot acts as the output. There is an insulation gap between the input and output section of $1.2 \mathrm{~mm}$. The generated magnetic field due to converse ME effect interacts with the external applied magnetic field producing flux gradient, which is detected through the frequency shift and output voltage change in gradiometer structure. The measurements of output voltage dependence on applied magnetic field clearly illustrate that the proposed design can provide high sensitivity and bandwidth. (c) 2010 American Institute of Physics. [doi:10.1063/1.3354985]
\end{abstract}

\section{INTRODUCTION}

Magnetoelectric (ME) composites use product property that involves induction of strain in the magnetostrictive material on application of magnetic field, which is then transferred to surrounding piezoelectric material resulting in generation of charge or voltage. The figure of merit of ME effect can be given by ME susceptibility $\alpha_{i j}$, which is a function of product of dielectric permittivity $(\varepsilon)$ and magnetic permeability $(\mu)$. Hence, a ME composite consisting of a piezoelectric material with high dielectric constant and a magnetic material with high permeability will have high interaction between the two phases resulting from the high degree of stress transfer. ${ }^{1-3}$

ME gradiometer detects gradient in magnetic field as a function of frequency near electromechanical resonance (EMR). Previously, we have proposed a gradiometer design based upon $0.8 \mathrm{~Pb}\left(\mathrm{Zr}_{0.52} \mathrm{Ti}_{0.48}\right) \mathrm{O}_{3}-0.2 \mathrm{~Pb}\left(\mathrm{Zn}_{1 / 3} \mathrm{Nb}_{2 / 3}\right) \mathrm{O}_{3}$ (PZT)-Terfenol-D laminate composite structure with unipoled transformer architecture. ${ }^{4}$ The fabricated gradiometer exhibited high sensitivity for detecting differential magnetic field near EMR. In this study, we fabricated two different designs of gradiometer with metal (nickel) and ceramic (PZT) as the material layers in the laminate composite. The dielectric permittivity $\left(\varepsilon_{r}\right)$ of the PZT was calculated to be 1290. Nickel was selected over other materials considering its cofiring ability with PZT, which will allow fabrication of multilayer structures using well established tape-casting technique. Nickel is widely used as conductive paste in multilayer chip capacitors owing to its high conductivity and low cost. It has been found that when nickel is fabricated into nanosized powder, it forms chainlike structures due to

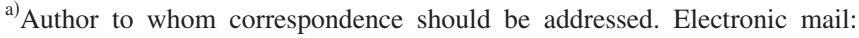
spriya@vt.edu.
}

its magnetic properties. The chain-form nickel powder based conductive paste has eight times higher conductivity than that fabricated from conventional powders. ${ }^{5}$ Thus, it is possible to utilize both the characteristics of nickel at the same time, namely, magnetostriction and electrical conductivity. Nickel and cobalt exhibit excellent magnetostrictive properties. The values of saturation magnetostriction $\lambda_{s}$ for $\mathrm{Ni}$ and Co are lower than $\mathrm{CoFe}_{2} \mathrm{O}_{4}$ but are comparable to some common ferrites such as $\mathrm{NiFe}_{2} \mathrm{O}_{4}$ and $\mathrm{MnFe}_{2} \mathrm{O}_{4}$. The magnitude of magnetostriction in rare-earth iron alloys is significantly higher but they suffer from the drawback of poor electrical properties, complex synthesis, and incompatibility in cosynthesis with PZT. Ni exhibits anisotropic magnetostriction $\left(\lambda_{111}=-25 \times 10^{-6}\right.$ and $\left.\lambda_{100}=-46 \times 10^{-6}\right)$ with an easy direction along $\langle 111\rangle$. The electrical resistivity of $\mathrm{Ni}$ $\left(\sim 6 \times 10^{-6} \Omega^{-1} \mathrm{~cm}^{-1}\right)$ is comparable to that of Co $\left(\sim 5.5 \times 10^{-6} \Omega^{-1} \mathrm{~cm}^{-1}\right)$, which allows both of them to be suitable for conductors. ${ }^{6}$ It is possible to modify the magnetocrystalline properties of metal particles by processing conditions leading to changes in microstructure, morphology, surface chemistry, and particle shape. Nickel is a magnetically soft material and has a saturation dc magnetic field on the order of few hundreds of oersteds. Hence, in order to investigate the shift in resonance frequency of the gradiometer two different magnetic dc bias ranges were selected. The first magnetic dc bias range of 0-100 Oe was chosen to understand the effect of coercivity on the gradiometer characteristics and the second magnetic dc bias range of 0-1600 Oe was selected to evaluate the performance in the full range.

\section{EXPERIMENTAL}

The ME gradiometer sample A (design 1) had the ringdot electrode pattern printed on top and bottom surfaces of PZT, where the ring acts as the input while the dot acts as the 


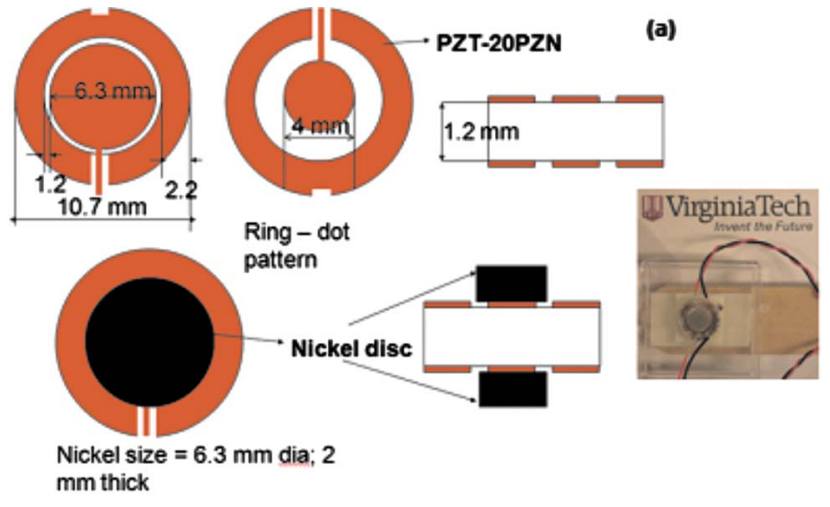

TABLE II. Equivalent circuit parameter of the input and output sections at resonance-antiresonance frequency range.

\begin{tabular}{lcc}
\hline \hline Sample A & Input section & Output section \\
\hline$R(\mathrm{k} \Omega)$ & 1.71 & 1.67 \\
$L(\mathrm{mH})$ & 23.085 & 48.97 \\
$C_{a}(\mathrm{pF})$ & 16.785 & 8.392 \\
$C_{b}(\mathrm{pF})$ & 467.235 & 315.06 \\
& & \\
Sample B & & \\
$R(\Omega)$ & 337.94 & 669.56 \\
$L(\mathrm{mH})$ & 7.31 & 14.37 \\
$C_{a}(\mathrm{pF})$ & 62.75 & 32.485 \\
$C_{b}(\mathrm{pF})$ & 719.39 & 307.49 \\
\hline \hline
\end{tabular}

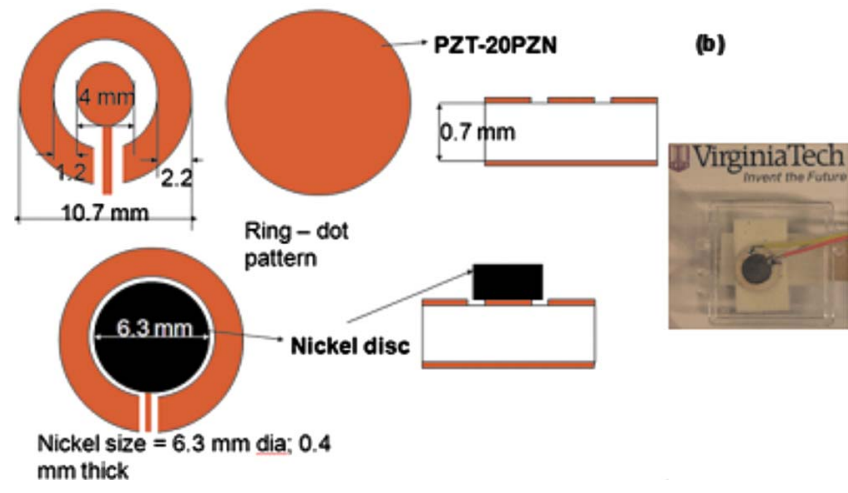

FIG. 1. (Color online) (a) Gradiometer design 1 (sample A): fabrication of ring-dot electrodes with stripe electrode pattern and attachment of nickel disk on the output electrodes. Inset: picture of fabricated gradiometer. (b) Gradiometer design 2 (sample B): ring-dot electrode pattern with common ground electrode and attachment of nickel disk on the output electrode. Inset: picture of fabricated gradiometer.

output. The output section had a diameter of $4 \mathrm{~mm}$ and the input section had a width of $2.2 \mathrm{~mm}$. There is an insulation gap between the input and output sections of $1.2 \mathrm{~mm}$. Two nickel disks with diameter of $6.3 \mathrm{~mm}$ and thickness of $2 \mathrm{~mm}$ were bonded on the output section, as shown in Fig. 1(a). The inset shows a picture of fabricated gradiometer. Thus, the gradiometer had separated ground electrode which avoids cross-talk between the input and output sections. The gradiometer sample B (design 2) also had the ring-dot electrode pattern printed on the top surface of PZT, where the ring acts as the input while the dot acts as the output. The output section had a diameter of $6.3 \mathrm{~mm}$ and the input section had a width of $2.2 \mathrm{~mm}$. There is an insulation gap between the input and output sections of $1.2 \mathrm{~mm}$. The ground electrode was at the bottom of the PZT. Nickel disk with diameter of $6.3 \mathrm{~mm}$ was bonded on the output section, as shown in Fig. 1(b), where the inset shows the picture of fabricated gradiometer. Thus, in this case the gradiometer had a common ground electrode which is easier to fabricate. In both constructions, ultracorrosion-resistant nickel (alloy 200/201) was used with the elemental composition shown in Table I.

The piezoelectric constant of the synthesized composites was measured by APC YE 2730A d 33 meter. Resonance characteristics were determined by HP 4194A impedance analyzer (Hewlett Packard Co., USA). For the gradiometer samples $\mathrm{A}$ and $\mathrm{B}$, the input voltage of $10 \mathrm{~V}_{\mathrm{p}-\mathrm{p}}$ was applied on the ring section and the output voltage was measured from the dot section using a Tektronix TDS 420A four channel digital oscilloscope with varying dc magnetic bias field. The frequency range was chosen near resonance frequency of the transformer, ${ }^{7-9}$ i.e., $249 \mathrm{kHz}$ for sample A and $235 \mathrm{kHz}$ for sample B. The magnetic field generated in the dot section was measured by using the magnetic field sensor (microprocessor controlled precision gauss meter from Walker LDJ Scientific, Inc.). All the measurements were repeated to check the consistency of results.

\section{RESULTS AND DISCUSSION}

For a piezoelectric transformer operating near the resonance point, the equivalent circuit can be represented, as shown in Table II. In this circuit $R, L$, and $C_{a}$ are the motional resistance, inductance, and capacitance, and $C_{b}$ is the damped capacitance. Figures 2(a)-2(d) show the open circuit (OC) and short circuit (SC) impedance responses of sample A and sample B, respectively. Using these spectra, the magnitude of equivalent circuit parameters was calculated for both input and output. The matching impedance $\left(Z_{\text {match }}\right)$ of a piezoelectric transformer is given as

TABLE I. Elemental composition of the nickel alloy used in the fabrication of the gradiometer.

Nickel 200/201 chemical composition

$(\%)$

\begin{tabular}{lccccccc}
\hline & Ni $(\min )$ & $\mathrm{Fe}(\max )$ & $\mathrm{Cu}(\max )$ & $\mathrm{C}(\max )$ & $\mathrm{Mn}(\max )$ & $\mathrm{S}(\max )$ & $\mathrm{Si}(\max )$ \\
\hline Nickel 200 & 99.0 & 0.40 & 0.25 & 0.15 & 0.35 & 0.01 & 0.35 \\
Nickel 201 & 99.0 & 0.40 & 0.25 & 0.02 & 0.35 & 0.01 & 0.35 \\
\hline \hline
\end{tabular}



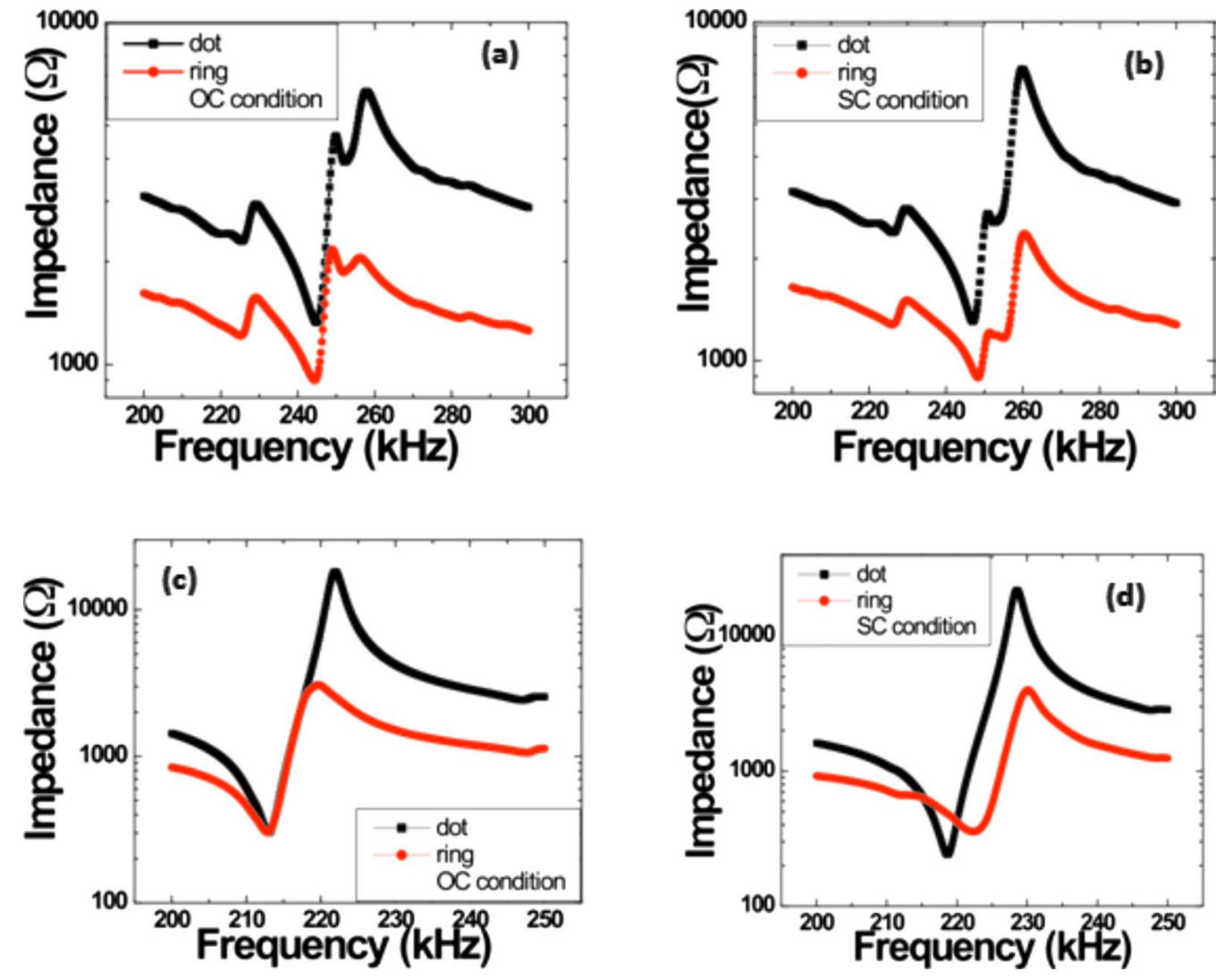

FIG. 2. (Color online) (a) OC and (b) SC impedance responses of sample A. (c) OC and (d) SC impedance responses of sample B.

$$
Z_{\text {match }}=\frac{1}{2 \pi f_{r} C_{b(\text { output })_{\text {input short }}}},
$$

where $f_{r}$ is the resonance frequency. Using the values listed in Table II, the matching impedance was calculated to be $2028 \Omega$ for sample A and $2202 \Omega$ for sample B. Simplistically, the gain of the transformer is given by

$$
\text { Gain }=\sqrt{\frac{C_{a(\text { input })}}{C_{a(\text { output })}}} .
$$

Here, the transformer gains are 1.414 for sample A and 1.39 for sample B. The gain of the transformer structure affects the resonance frequency shift and it can be improved by using the multilayer structure in the input section. Since the capacitance is inversely proportional to the thickness of each layer, one can enhance the gain significantly by fabricating very thin layers with interdigital electrode pattern in the input section. The efficiency of the transformer is given by

$$
\text { Efficiency }=\frac{1}{1+4 \pi f_{r, \text { in }} C_{a, \text { in }} R_{\text {in }}\left(\frac{C_{b, \text { out }}}{C_{a, \text { out }}}\right)},
$$

where in and out stand for the input and output, respectively. The expected efficiency of the transformer for sample A and sample B was calculated to be $99.8 \%$. The efficiency of the transformers is expected to be similar due to the similar dimensions of the input and output sections. As mentioned earlier, the transformer shows high sensitivity near the EMR frequency range. This range can be determined by noticing that the transformer is always operated between the frequency limits given by

$$
\omega_{\mathrm{SC}}=\frac{1}{\sqrt{L C}}
$$

and

$$
\omega_{\mathrm{OC}}=\frac{1}{\sqrt{L\left(C^{-1}+C_{d, \mathrm{out}}^{-1}\right)^{-1}}},
$$

where $\omega$ refers to the angular frequency. The operating frequency range for sample A was calculated based on Eqs. (4) and (5) as 248.2-251.5 kHz and that for sample B as 232.9$244.9 \mathrm{kHz}$. From Eqs. (1)-(5), it can be seen that the transformer characteristics for a unipoled structure are directly related to the ratio of input/output area. Higher area ratio means higher capacitance ratio of input and output sections and vice versa. The transformer gain varies directly with area ratio. For high area ratio, large gain can be obtained. In order to increase the sensitivity of the gradiometer, higher voltage gain is essential, which can be obtained with higher inputoutput area ratio of the transformer architecture. Higher gain will generate higher output voltage in the output section, thereby increasing the ability of the ME gradiometer to quantify magnetic field gradient near EMR range. The most favorable condition for the transformer action in unipoled case is when the impedance spectrum of the input and output sections is matching. This means that the difference between the resonance frequencies of the output and input in the $\mathrm{OC}$ 
condition and the difference between the antiresonance frequencies between the output and input sections in the SC condition are equal. In this condition, both the input and output sections resonate at the same frequency which allows the effective conversion of energy. The effective coupling factor given by $k^{2}=f_{a}^{2}-f_{r}^{2} / f_{a}^{2}$ is maximum in this condition. It should be mentioned here that both intrinsic and extrinsic losses play an important role at the resonance. Specifically, mechanical losses become a dominant factor in achieving sharp resonance peaks and should be minimized. Tradition- ally, dopants such as Mn and Co have been used to reduce the magnitude of the mechanical losses. We did not consider loss minimization in this study but it should be incorporated in the future to further improve the performance.

The working principle of ME sensor is based on measuring the variation in ME voltage coefficient due to changes in applied magnetic field. The response of sample to the external magnetic field is related to the magnitude of ME voltage coefficient. The description of ME effect for the gradiometer at the EMR frequency range is given by the theory as ${ }^{10-12}$

$$
\alpha_{E}=-\frac{2(1+\nu)(1-V)^{p} s_{11} J_{1}(k R)^{p} d_{31}^{m} q_{31}}{(1-\nu)^{p} s_{11}\left[a J_{0}(k R)-(1-\nu) s_{1} J_{1}(k R)\right]^{p} \varepsilon_{33}+2\left[a J_{0}(k R)-s_{3} J_{1}(k R)\right]^{p} d_{31}^{2}},
$$

where $a=k R s_{1}, s_{1}=V^{m} s_{11}+(1-V)^{p} s_{11}, s_{3}=(1-\nu)(1-V)^{p} s_{11}$ $+2 V^{m} s_{11}$

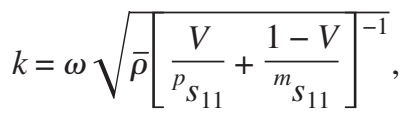

$\bar{\rho}=V^{p} \rho+(1-V)^{m} \rho$ is the average density of the composite material, $\omega$ is the angular frequency, $q_{i j}$ and $d_{i j}$ are piezo- magnetic and piezoelectric coefficients, $\varepsilon_{i j}$ is the permittivity matrix, $R$ is the radius, $V$ is the piezoelectric volume fraction, Poison's coefficient ${ }^{m} \nu$ is assumed to equal ${ }^{p} \nu=\nu$ for simplicity, and $J_{0}$ and $J_{1}$ are Bessel functions of the first kind. The shift in the EMR frequency range for Ni-PZT gradiometer was derived by using the theory described in our earlier work and taking into account the material parameters of
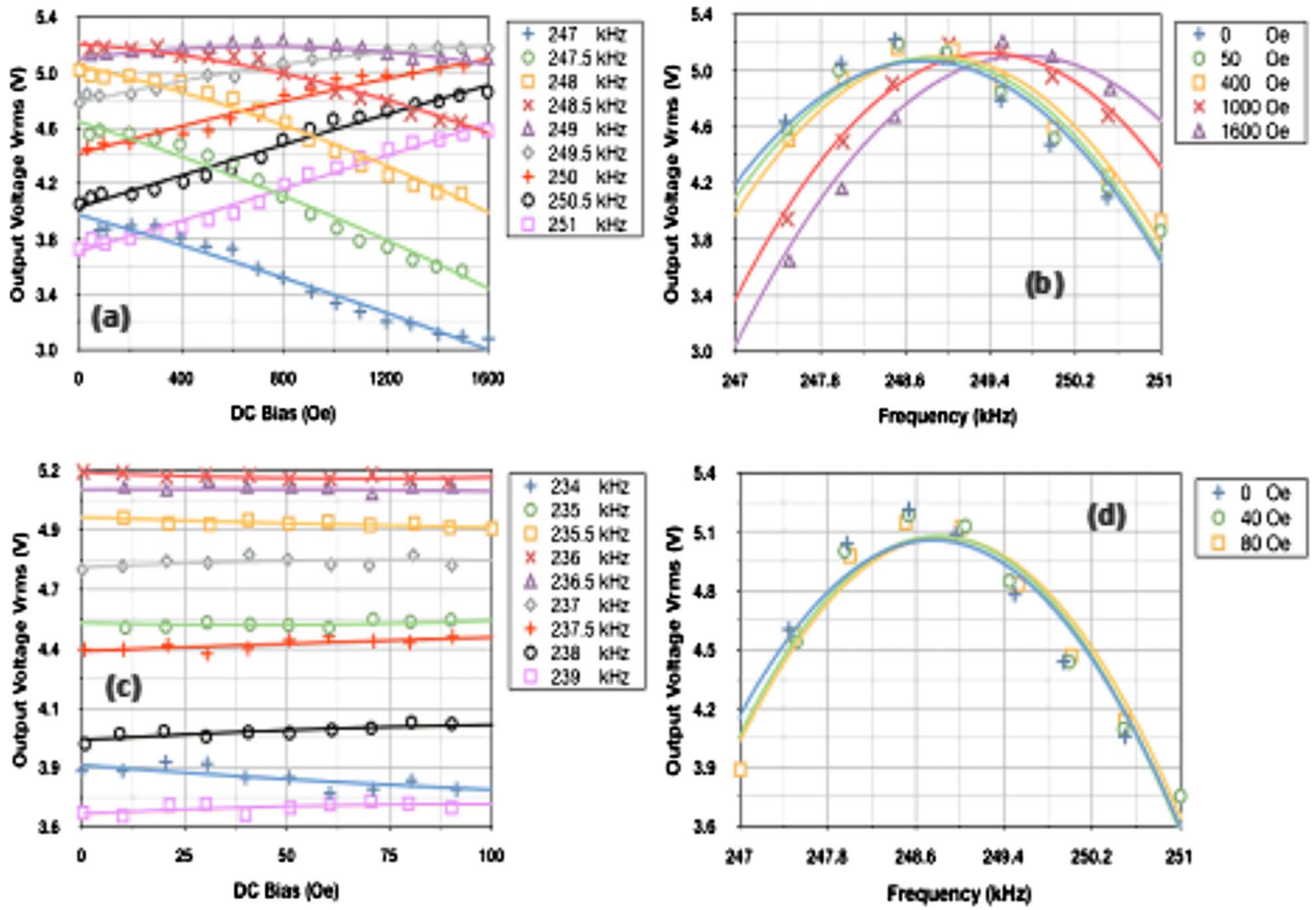

FIG. 3. (Color online) Theoretical dependence (solid line) and experimental results (symbols) of ring-dot gradiometer design 1 (sample A). (a) Output voltage as a function of dc magnetic field $(0-1600 \mathrm{Oe})$ at varying frequencies. (b) Output voltage as a function of frequency at varying dc magnetic field (0-1600 Oe). (c) Output voltage as a function of dc magnetic field (0-100 Oe) at varying frequencies. (d) Output voltage as a function of frequency at varying dc magnetic field $(0-100 \mathrm{Oe})$. The input voltage of the gradiometer was fixed at $10 \mathrm{~V}_{\mathrm{p}-\mathrm{p}}$. 

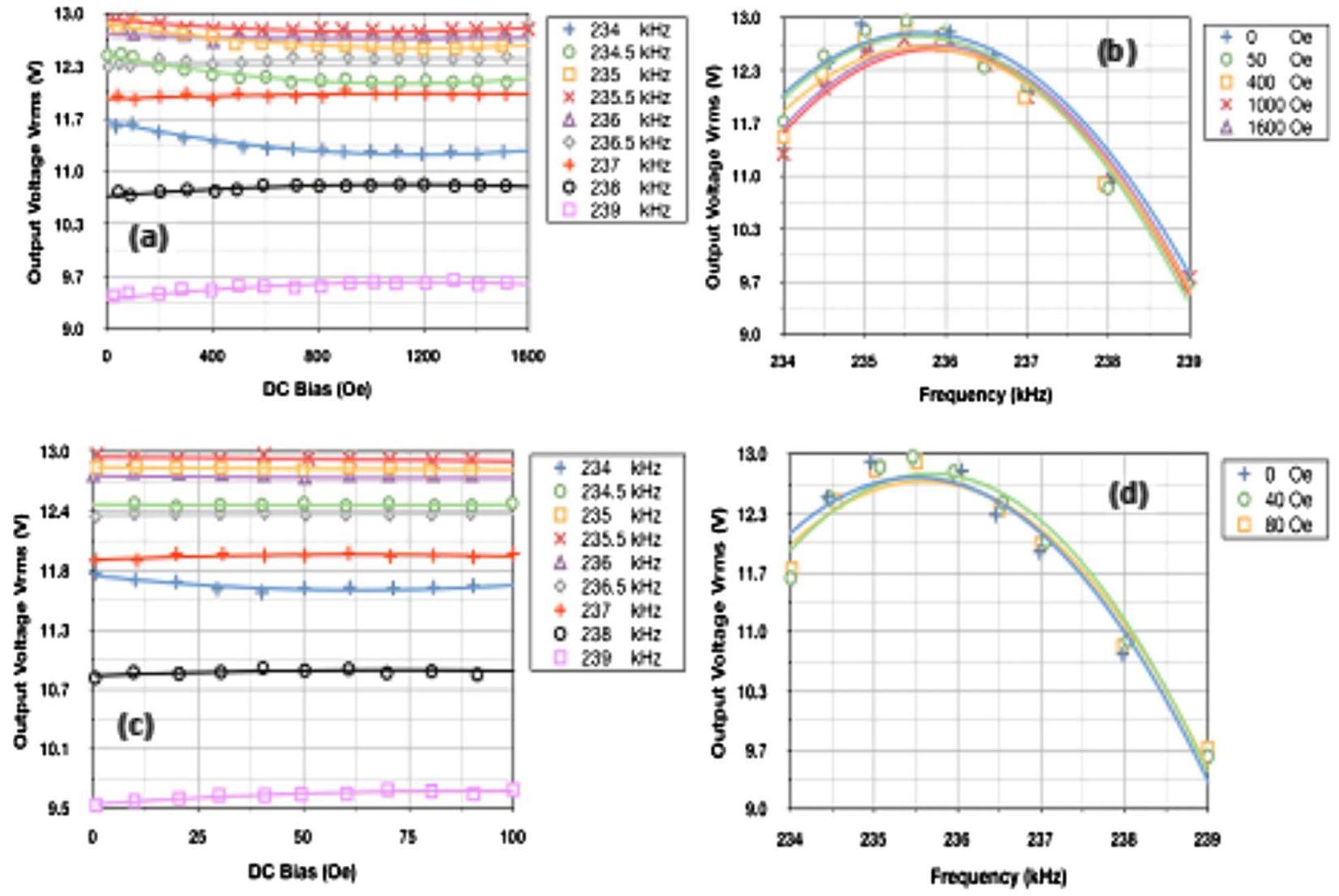

FIG. 4. (Color online) Ring-dot gradiometer design 1 (sample B). (a) Output voltage as a function of dc magnetic field (0-1600 Oe) at varying frequencies. (b) Output voltage as a function of frequency at varying dc magnetic field (0-1600 Oe). (c) Output voltage as a function of dc magnetic field (0-100 Oe) at varying frequencies. (d) Output voltage as a function of frequency at varying dc magnetic field ( $0-100$ Oe). The input voltage of the gradiometer was fixed at $10 \mathrm{~V}_{\mathrm{p}-\mathrm{p}}$.

nickel. ${ }^{4,10-12}$ Material parameters for nickel taken in the calculation were $\rho=8900 \mathrm{~kg} / \mathrm{m}^{3}$, Young's modulus $=20$ $\times 10^{10} \mathrm{~N} / \mathrm{m}^{2}, \mu_{33} / \mu_{0}=20$, and $q=57 \times 10^{-6} \mathrm{Oe}^{-1}$. The material parameters for PZT taken in calculation were $\rho$ $=7300 \mathrm{~kg} / \mathrm{m}^{3}$, Young's modulus $=0.7 \times 10^{11} \mathrm{~N} / \mathrm{m}^{2}, \varepsilon_{33} / \varepsilon_{0}$ $=4100$, and $d=289 \times 10^{-12} \mathrm{C} / \mathrm{N}$.

Figure 3(a) shows the variation in output voltage in the dot section of sample A with dc bias field for various frequencies near EMR. Figure 3(b) shows the variation in output voltage with frequency for different applied dc magnetic fields. Figures 3(c) and 3(d) show the variation in output voltage generated in the dot section as a function of dc bias field and frequency in the low dc bias field of 0-100 Oe. In Fig. 3, the solid line represents the theoretical values while the experimental data are shown by dots. The input voltage on the ring section was maintained at $10 \mathrm{~V}_{\mathrm{p}-\mathrm{p} \text {. It can be seen }}$ in Fig. 3(a) that there is change in slope on either side of EMR. At the frequency of 250 and $250.5 \mathrm{kHz}$, the variation in output voltage is linear at higher applied magnetic field. In Fig. 3(b), two regions can be clearly separated and the device can provide differential voltage with frequency as the magnetic field changes with respect to reference state. The sensitivity of sensor is determined by the smallest dc field at which it can provide a large differential change. In the region of $0-100$ Oe, there is no crossover or change in the slope of the lines around EMR. Similarly, there is no obvious shift in the peak of the output voltage as a function of frequency. Thus, the fields required for the nickel-PZT gradiometer are higher than 100 Oe.

Figure 4(a) shows the variation in output voltage in the dot section of sample B as a function of dc bias field for various frequencies near EMR, whereas Fig. 4(b) shows the variation in output voltage as a function of frequency for different applied dc magnetic fields. Comparing these data with that of sample A in Fig. 3, it can be seen that the variation in slope of the output voltage is small even at high fields. Further, there is very small shift in the peak of output voltage near the EMR. Similarly, Figs. 4(c) and 4(d) show the output voltage generated in the dot section of sample B as a function of dc bias field and frequency for the dc bias range of 0-100 Oe. In all these measurements, the input voltage on ring section was maintained at $10 \mathrm{~V}_{\mathrm{p}-\mathrm{p}}$. Using these results, it can be summarized that sample A shows a better performance. We can expect that separated electrode pattern reduces the noise level and interference between the input and output signals. Further improvement in the response of sample A can be obtained by increasing the insulation gap, increasing the gain, and magnetically shielding the input section.

Figures 5(a)-5(d) show the differential voltage change as a function frequency at constant dc magnetic fields with respect to reference state for two different ranges. It can be 

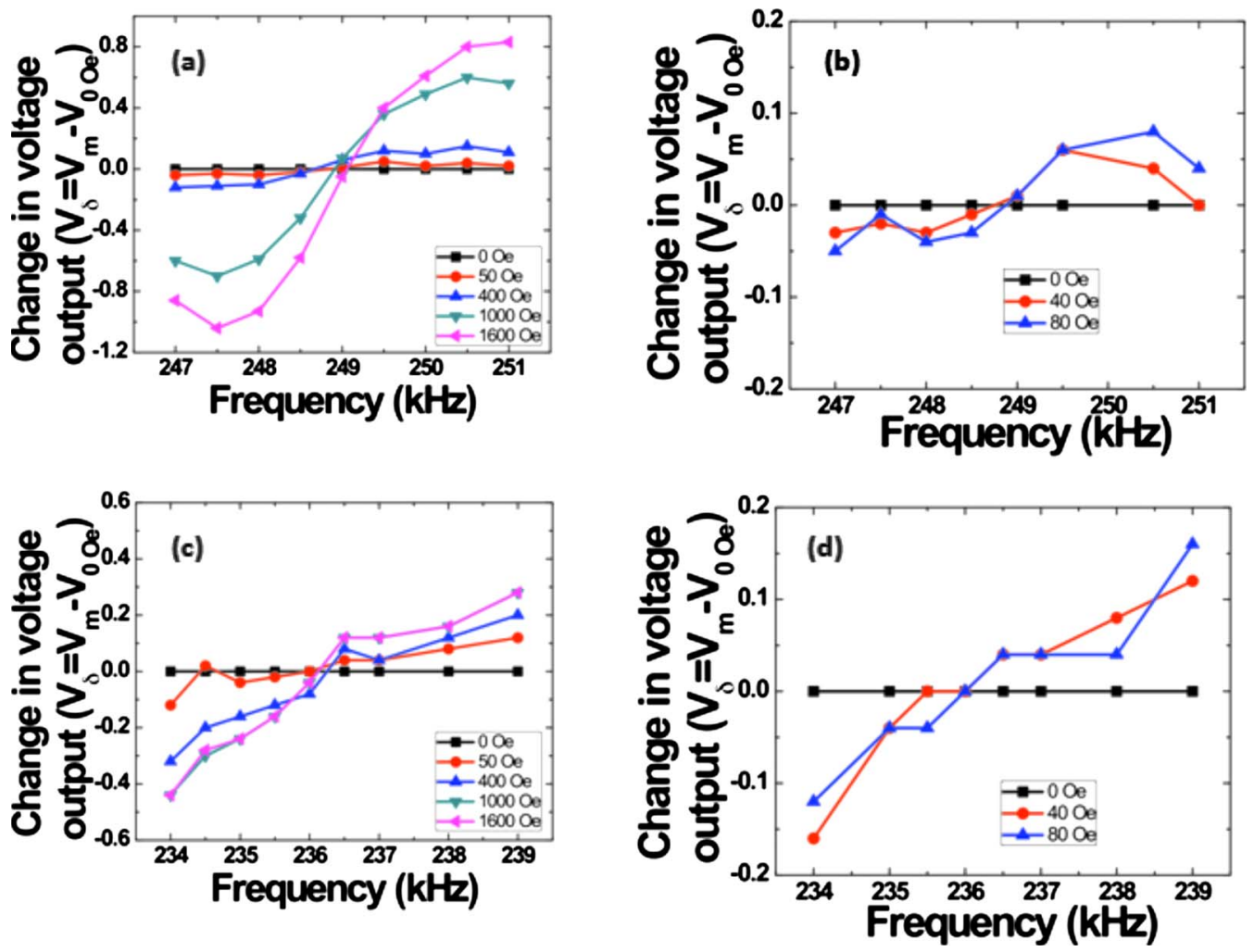

FIG. 5. (Color online) Change in output voltage with respect to change in the dc magnetic field. Input was fixed at $10 \mathrm{~V}_{\mathrm{p}-\mathrm{p}}$ for both sample $\mathrm{A}$ and sample $\mathrm{B}$. (a) Differential change in the dc bias range of 0-1600 Oe for sample A. (b) Differential change in the dc bias range of 0-100 Oe for sample A. (c) Differential change in the output voltage with respect to change in the dc bias for sample B. (d) Differential change in the dc bias range of 0-100 Oe for sample B.

noted in Fig. 5(a) that the change in the output voltage increases as dc bias field increases. Above 1000 Oe, there is a significant voltage change of $\sim 1 \mathrm{~V}$ across the resonance frequency. In the frequency range of $248-250 \mathrm{kHz}$, there is almost a linear variation in the differential voltage change for applied dc bias of 1000 Oe and higher. For lower dc bias, there is no clear trend seen in the variation in differential voltage change. This indicates that for sample A, the sensitivity of the gradiometer is higher than 1000 Oe with bandwidth in the range of $2 \mathrm{kHz}$. We think this is quite a good performance for single layer metal-ceramic gradiometer. The performance can be easily improved by multilayering the input section of the gradiometer. In case of sample B, similar changes can be seen, i.e., linear variation in differential output voltage as a function of frequency at high dc bias, but the response is noisy which can be correlated with the cross-talk between the input and output sections. Sample B generates higher output voltage in the dot section; however, sample A shows higher sensitivity for detecting gradient magnetic field since it has two input and two output sections which allow transfer of stress on both sides of the PZT resulting in higher overall change in the differential magnetic field. This clearly shows that the separated ground electrode section provides a clean voltage spectrum. The results of Figs. 3 and 5 show that sample A with one layer of PZT and nickel can provide the capability of measuring gradient in magnetic field above 1000 Oe.

\section{SUMMARY}

The results of this study show that piezoelectric unipoled transformer with ME laminate structure in the output section can provide magnetic field gradient measuring capability. A linear change in the output voltage as a function of dc bias field was observed near EMR. The output voltage measured in the dot section of the gradiometer was found to increase linearly with increasing input voltage in the ring section. It was found that Ni-PZT gradiometers work in higher frequency ranges, $247-251 \mathrm{kHz}$ for sample A and $234-239 \mathrm{kHz}$ for sample B, maintaining the sensitivity of the gradiometer over a wide range near EMR frequency. The bandwidth for sample A was found to be more than $2 \mathrm{kHz}$ at magnetic dc bias of higher than 1000 Oe. The metal-ceramic structure reported here is extremely a cost-effective technology for designing gradient magnetic field sensors and its performance can be easily improved by incorporating multilayering process in the input section.

\section{ACKNOWLEDGMENTS}

The authors gratefully acknowledge the financial support from DMR-Metals, National Science Foundation, and Air Force Office of Scientific Research.

${ }^{1}$ S. Priya, R. Islam, S. Dong, and D. Vieland, J. Electroceram. 19, 147 (2007).

${ }^{2}$ C. W. Nan, M. I. Bichurin, S. Dong, D. Vieland, and G. Srinivasan, J. Appl. Phys. 103, 031101 (2008).

${ }^{3}$ M. Fiebig, J. Phys. D 38, R123 (2005).

${ }^{4}$ V. Bedekar, R. A. Islam, H. Kim, M. I. Bichurin, S. N. Ivanov, Y. J. Pukinski, and S. Priya, Eur. Phys. J. B 71, 387 (2009).

${ }^{5}$ Source website: http://www.sei.co.jp/news_e/press/01/01_22.html.

${ }^{6}$ R. E. Newnham, Properties of Materials: Anisotropy, Symmetry, Structure (Oxford University Press, New York, 2005).

${ }^{7}$ R. Islam, H. Kim, S. Priya, and H. Stephanou, Appl. Phys. Lett. 89, 152908 (2006). 
${ }^{8}$ H. Kim, R. Islam, and S. Priya, Appl. Phys. Lett. 90, 012909 (2007). ${ }^{9}$ S. Priya, S. Ural, H. Kim, K. Uchino, and T. Ezaki, Jpn. J. Appl. Phys., Part 1 43, 3503 (2004).

${ }^{10}$ M. I. Bichurin, V. M. Petrov, and G. Srinivasan, Phys. Rev. B 68, 054402 (2003).
${ }^{11}$ M. I. Bichurin, D. A. Filippov, V. M. Petrov, V. M. Laletsin, N. Paddubnaya, and G. Srinivasan, Phys. Rev. B 68, 132408 (2003).

${ }^{12}$ G. Srinivasan, C. P. De Vreugd, V. M. Laletin, N. Paddubnaya, M. I. Bichurin, V. M. Petrov, and D. A. Filippov, Phys. Rev. B 71, 184423 (2005) 